\title{
NHỮNG CHIẾN LƯợC XIN LỖI BẰNG TIẾNG ANH CỦA NGƯờI MĨ VÀ NGƯờI VIÊTT
}

\author{
Nguyễn Thùy Trang* \\ Khoa Ngôn ngũ và Văn hóa các nước nói tiếng Anh, Truờng Đại học Ngoại ngũu, ĐHQGHN, \\ Phạm Văn Đồng, Cầu Giá́y, Hà Nội, Việt Nam \\ Nhận bài ngày 14 tháng 2 năm 2017 \\ Chỉnh sửa ngày 23 tháng 3 năm 2017; Chấp nhận đăng ngày 24 tháng 3 năm 2017
}

Tóm tắt: Nghiên cứu này tìm hiểu chiến lược xin lỗi bằng tiếng Anh của 20 đối tượng khảo sát (ĐTKS) Việt và 20 ĐTKS Mĩ. Các ĐTKS Việt có kinh nghiệm làm việc với người Mĩ tại Việt Nam, và các ĐTKS Mĩ đang làm việc tại Việt Nam. Dữ liệu được thu thập qua phiếu câu hỏi diễn ngôn gồm ba tình huống đã chỉ ra một số tương đồng và khác biệt chủ yếu trong việc lựa chọn và sử dụng các chiến lược xin lỗi (CLXL) của hai nhóm dựa trên danh mục CLXL trong nghiên cứu của Cohen và Olshtain (1993) và Trosborg (1995). Kết quả nghiên cứu hỗ trợ lập luận của các nghiên cứu giao văn hóa trước đây của Olshtain (1989) và Elli (1994) rằng việc sử dụng các CLXL ở các nền văn hóa khác nhau có xu hướng tương đồng về mặt ngôn ngữ bất kể khác biệt về bối cảnh và mức độ nghiêm trọng. Điểm khác biệt cơ bản là trong khi người Việt thiên về chiến lược bày tỏ quan tâm đến đồng nghiệp khi xin lỗi thì phần lớn ĐTKS Mĩ ưu tiên giải quyết lỗi theo chiến lược đề nghị đền bù, thông qua đó đặc trưng của một nền văn hóa Việt trọng tình cảm và tính tập thể cao cũng như một nền văn hóa Mĩ thiên về lý trí và trọng cá nhân được phản ánh rõ nét. Kết quả cũng cho thấy việc tiếp xúc liên văn hóa với đồng nghiệp không ảnh hưởng đến các CLXL mà nhóm người Việt và người Mĩ sử dụng trong nghiên cứu này. ${ }^{(1)}$

Từ khóa: chiến lược xin lỗi, văn hóa Mĩ, văn hóa Việt, hành vi lời nói

\section{Phần mở đầu}

Chính sách mở cửa cùng với nền kinh tế toàn cầu ngày càng phát triển thúc đẩy cơ hội giao tiếp liên văn hóa của công dân trên toàn thế giới. Mặc dù được đánh giá là những người học ngoại ngữ nắm rất chắc hệ thống ngữ pháp tiếng Anh, không nhiều trong số chúng ta dám đảm bảo rằng ta có thể giao tiếp thành thạo với người khác khi sử dụng tiếng Anh như một ngoại ngữ. Geertz (1973) hay Trice và Beyer (1992) đã cho rằng giao tiếp liên văn hóa đầy thử thách vì cái mà ta coi là

* ĐT.: 84-985081325, Email: trangnt1912@gmail.com ${ }^{1}$ Nghiên cứu này được hoàn thành với sự hỗ trợ của Trường Đại học Ngoại ngữ, Đại học Quốc gia Hà Nội trong đề tài mã số N.16.03 văn hóa của mình lại có thể là " một hệ thống các ý nghĩa biểu tượng ngoại đạo" với một hay nhiều nhóm người khác. Thực ra, sự khác biệt trong quy tắc sử dụng ngôn ngữ trong xã hội gây ra nhiều trở ngại cho người học ngoại ngữ và thậm chí dẫn đến nhiều hiểu lầm trong giao tiếp. Đã có nhiều học giả quan tâm và tìm hiểu sâu về khía cạnh này trong các bối cảnh giao tiếp liên văn hóa khác nhau. Các nghiên cứu về hành động lời nói (speech act) đã chỉ ra rằng cách xin lỗi là một hành vi lời nói (HVLN) nhạy cảm bởi trên thế giới này, khi các quy ước ứng xử xã hội khác biệt dù ít dù nhiều thì cách xin lỗi trong các bối cảnh khác nhau cũng không thể tương đồng, đó là điều khá hiển nhiên. 
Trong lịch sử, một số các nghiên cứu về hành vi xin lỗi (HVXL) được thực hiện bởi Cohen, Olshtain và Rosenstein (1986), García (1989), Trosborg (1987, 1995), Bergman và Kasper (1993), Maeshiba, Yoshinaga, Kasper, \& Ross (1996) hay Rose (2000) cho thấy khi thực hiện HVXL bằng tiếng Anh, các đối tượng khảo sát (ĐTKS) đều chịu ảnh hưởng nhất định của ngôn ngữ mẹ đẻ, và có sự khác biệt trong việc sử dụng các chiến lược xin lỗi (CLXL) so với cách người bản địa thực hiện HVLN này. Nghiên cứu nổi bật nhất không thể bỏ qua là nghiên cứu "Lời yêu cầu và lời xin lỗi trong giao thoa ngữ dụng học" được thực hiện tại bảy vùng lãnh thổ khác nhau bởi tác giả Blum-Kulka và các cộng sự. Tại Việt Nam, một số tác giả cũng thực hiện điều tra các HVLN theo phương pháp tương phản ngữ dụng truyền thống trong ngôn ngữ Việt đối với ngôn ngữ Anh đối với cách chào hỏi (Nguyễn Phương Sửu, 1990), cách khen và đáp lại lời khen (Nguyễn Quang, 1998), cách yêu cầu và tiếp đáp yêu cầu (Đỗ Thị Mai Thanh, 2000; Phan Thị Vân Quyên, 2001), hay cách nói không đồng tình (Kiều Thị Thu Hương, 2006). Cho đến nay, một số nghiên cứu riêng lẻ ở cấp khóa luận cử nhân và luận văn cao học về HVXL cũng được nghiên cứu theo hướng này. Ví dụ, trong nghiên cứu của Đặng Thanh Phương (2000), tác giả kết luận rằng lời xin lỗi trong tiếng Anh và tiếng Việt khác nhau về mức độ thẳng thắn và tính gián tiếp tùy thuộc vào bối cảnh giao tiếp, và lời xin lỗi của nhóm đối tượng Anh sử dụng nhiều dấu hiệu từ vựng (lexical markers) trong khi nhóm người Việt sử dụng nhiều dấu mốc lịch sự (politeness markers) trong lời xin lỗi và phúc đáp hơn. Nghiên cứu của Kiều Thị Hồng Vân (2000) nhấn mạnh vào các yếu tố ảnh hưởng chính đến các chiến lược xin lỗi
(CLXL) của hai nhóm người Việt và người Anh, và kết luận rằng độ tuổi có tác động lớn nhất đến cách xin lỗi của người Việt trong khi khoảng cách quyền lực và mức độ thân quen có ảnh hưởng nhiều đến việc lựa chọn CLXL của nhóm người Anh. Một số nghiên cứu trong đó ĐTKS thực hiện HVXL sử dụng tiếng Anh như một ngoại ngữ cũng được thực hiện trên một vài nhóm đến từ các nền văn hóa khác (Bergman \& Kasper, 1993; Rose, 2000; Trosborg, 1987, 1995). HVXL được xem là một khía cạnh nhạy cảm nhưng rất phổ biến trong đời sống thường ngày, tuy nhiên lại chưa nhận được sự quan tâm ở Việt Nam. Vì vậy, nghiên cứu này được tiến hành nhằm tìm hiểu về các CLXL mà hai nhóm đối tượng sử dụng, từ đó tìm ra những tương đồng và khác biệt trong việc lựa chọn CLXL của họ cũng như xem xét các yếu tố tác động đến việc lựa chọn CLXL của hai nhóm đối tượng, và ảnh hưởng của môi trường làm việc và việc giao tiếp hàng ngày tại nơi làm việc lên cách xin lỗi của hai nhóm.

\section{Cơ sở lý luận}

\subsection{Văn hóa giao tiếp Việt và văn hóa giao tiếp $M \tilde{\imath}$}

Trong giao tiếp, người Việt được coi là nhóm người coi trọng tính tập thể và bối cảnh giao tiếp (Nguyễn Quang, 1998; Gorlanes \& Brilhart, 1997; Smith, Esmond \& Pham, 1996). Cách giao tiếp của họ thiên về hướng gián tiếp, mang nhiều ẩn ý và có xu hướng ưu tiên duy trì "việc làm vừa lòng nhau trong ứng xử với cộng đồng (Gudykunst, Matsumoto, Ting-Toomey, Nishida, Kim, \& Heyman, 1996). Ngoài ra, người Việt xử lý các tình huống trong xã hội thiên về tình hơn lý (Mạc Giao, 2002), nói cách khác, con người trong xã hội trọng tình cảm và lòng biết ơn, và hướng 
tới tính hòa thuận trong giao tiếp cộng đồng. Người Việt luôn đặt thể diện và lợi ích của cá nhân trong thể diện của tập thể (Hofstede, 1980), vì vậy Mạc Giao lý giải rằng trong các tình huống một cá nhân gây lỗi, các thành viên khác trong gia đình từ cha mẹ, anh chị em ruột đến những người thân và ông bà tổ tiên trong họ tộc đều có thể bị liên quan.

Trái lại, nền văn hóa Mỹ theo Hall (1976) mang tính chất chu cảnh thấp. Cụ thể hơn, người Mỹ có xu hướng tôn vinh tính cá nhân, và việc giao tiếp không bị chi phối quá mạnh bởi khoảng cách quyền lực giữa các cá nhân trong xã hội (Stella, 1992). Có thể nói đây là nền văn hóa nhấn mạnh tính bình đẳng, và các tình huống trong xã hội được xử lý dựa trên lý lẽ thay vì tình cảm cá nhân. Trong tác phẩm của Wanning, (1999, p.116) có đoạn viết "Chúng tôi là nền văn hóa của kiện tụng, và chúng tôi luôn tin rằng luôn phải có ai đó chịu trách nhiệm cho bất cứ việc gì xảy ra trong xã hội này" (xem thêm Lustig \& Koester, 2006). Nguyễn Quang (2006) nhận định đây là một nền văn hóa pháp trị trong đó các hành vi xã hội chủ yếu được thực hiện thông qua việc tuân thủ theo đạo đức và pháp luật.

\subsection{Hành vi xin lỗi trong thuyết hành động} lòi nói

HVXL được xếp vào nhóm hành vi biểu đạt (expressives) trong nhóm các hành động lời nói của Yule (1997). Ở đây có thể hiểu HVXL về cơ bản được thực hiện để biểu đạt trách nhiệm của người gây lỗi nhằm trấn an đối tượng giao tiếp ở một mức độ nào đó. Tương tự với các HVLN khác, HVXL là một quang phổ rộng không chỉ đáp ứng một mục đích giao tiếp đơn lẻ, nó được xem như hành vi mang lại thể diện cho người được xin lỗi, và đe dọa thể diện của người phải xin lỗi (Brown
\& Levinson, 1978). Theo Goffman (1971, xem thêm Kasper, 1996), lời xin lỗi có hai loại, (i) Lời xin lỗi lịch sự sử dụng để tăng tính lịch sự và thường đứng trước các HVLN khác trong giao tiếp xã hội, và (ii) Lời xin lỗi thực chất có mục đích khắc phục thiệt hại thực tế gây ra cho đối phương giao tiếp, bao gồm đề nghị bồi thường cả tổn hại tinh thần và vật chất. Nhiều học giả (Bergman \& Kasper, 1993; Cohen et al, 1986; García , 1989; Maeshiba et al, 1996; Rose, 2000; Trosborg, 1986, 1995) đồng tình rằng lời xin lỗi chính là một ví dụ điển hình của hành vi ứng xử lịch sự liên quan đến thể diện trong giao tiếp, và rằng các chiến lược chính được sử dụng trong HVXL đều được tìm thấy ở nhiều ngôn ngữ khác nhau, nhưng việc lựa chọn chiến lược nào hay kết hợp các chiến lược đó như thế nào lại phụ thuộc vào các bối cảnh giao tiếp cụ thể trong các nhóm văn hóa riêng biệt sử dụng các ngôn ngữ khác nhau.

\subsection{Tình huống yêu cầu HVXL trong văn hóa} Mỹ và văn hóa Việt

"Các vấn đề trong việc so sánh hành vi giao tiếp liên văn hóa" của Woflson, Marmor và Jones (1989) gợi ý một số tình huống trong giao tiếp hàng ngày mà người Mĩ bắt buộc phải thực hiện HVXL. Trong nghiên cứu đó, nhóm tác giả nhận thấy người Mĩ quan tâm đến nghĩa vụ cam kết với công việc cá nhân hoặc xã hội, nghĩa vụ tôn trọng tài sản của người khác, không gây thiệt hại hay khó chịu cho người khác và một số yêu cầu nhạy cảm khác. Tác giả Sugimoto (1998) nhận thấy thường người Mỹ chỉ xin lỗi cho những lỗi do chính họ hay cùng lắm là một vài người thân khác như người vợ, chồng, con cái hay thú cưng của họ gây ra. Trong văn hóa Việt, các nghiên cứu trước đây (Đặng Thanh Phương, 2000; Kiều Thị Hồng Vân, 2000) thực hiện khảo sát 
các tình huống dẫn tới HVXL kết luận rằng người Việt thường xin lỗi khi họ bị buộc tội đạo văn trong trường học, không hoàn thành nhiệm vụ được giao trong công việc, hoặc khi gây thiệt hại đến tài sản của người khác.

\subsection{Các chiến lược xin lỗi}

Nghiên cứu của Olshtain và Cohen (1993) tóm tắt năm CLXL chính, trong đó hai chiến lược (1) Biểu đạt lời xin lỗi (Expression of apology) và (2) Nhận trách nhiệm (Acknowledgment of responsibility) được sử dụng trong mọi hoàn cảnh, còn ba chiến lược (3) Giải thich lỗi (Explanation or account of the violation), (4) Đề nghị sưa lỗi (Offer of the repair), và (5) Hứa không tái phạm (Promise of forbearance) được sử dụng tùy thuộc vào từng tình huống cụ thể. Năm chiến lược này cũng đã được sử dụng làm nền tảng trong nghiên cứu của Blum-Kulka và cộng sự.

Trosborg (1995) trong nghiên cứu tập trung vào lời yêu cầu và lời xin lỗi liên văn hóa đã thiết lập một danh sách CLXL có nhiều điểm tương đồng với năm CLXL của Olshtain và Cohen nhưng mở rộng hơn. Tám chiến lược mà tác giả xây dựng bao gồm: (0) Phủ nhận gây ra lỗi (Opt-out), (1) Tối thiểu hóa lỗi (Minimization), (2) Nhận trách nhiệm (Acknowledgment of responsibility), (3) Giải thich lỗi (Explanation or Accoun), (4) Nói xin lối (Apologize), (5) Đề nghị sủa lối (Offer of repair),(6) Hứa không tái pham (Promise of forbearance), (7) Bày tỏ sụ quan tâm (Express the concern for the hearer)

Trong nghiên cứu này, tác giả sẽ sử dụng cả hai danh sách CLXL trên để mã hóa và tổng hợp các chiến lược được sử dụng bởi các ĐTKS, từ đó sẽ xây dựng một danh sách mới những CLXL được sử dụng trong ba tình huống nghiên cứu.

\section{Nội dung nghiên cứu}

\subsection{Câu hỏi nghiên cứu}

Nghiên cứu này tập trung chủ yếu vào hành vi xin lỗi bằng lời sử dụng tiếng Anh của hai nhóm đối tượng chính là (1) ĐTKS Mĩ làm việc tại Việt Nam và (2) ĐTKS Việt làm việc với người Mĩ tại Việt Nam. Mục đích chính là tìm ra những CLXL hai nhóm sử dụng khi xin lỗi bằng tiếng Anh, tiếng mẹ đẻ của nhóm người Mĩ và ngoại ngữ đối với nhóm người Việt, từ đó tìm hiểu những ẩn tàng văn hóa tác động lên sự lựa chọn CLXL của họ. Để thực hiện điều đó, nghiên cứu tập trung trả lời ba câu hỏi chính sau đây:

1. Những CLXL mà ĐTKS Mĩ sử dụng trong ba tình huống là gì?

2. Những CLXL mà ĐTKS Việt sử dụng trong ba tình huống là gì?

3. Có điểm gì tương đồng và khác biệt giữa cách sử dụng CLXL của hai nhóm?

\subsection{Phwơng pháp nghiên cúu}

Nghiên cứu này sử dụng cả phương pháp định tính và định lượng, dữ liệu được thu thập thông qua phiếu câu hỏi diễn ngôn (Discourse Completion Task/ DCT) và được mã hóa chi tiết và phân tích bằng phương pháp thống kê và diễn giải. Trọng tâm của phương pháp phân tích là so sánh dữ liệu nhằm tìm hiểu các khác biệt và tương đồng trong lời xin lỗi của hai nhóm đối tượng, như van de Vijver và Leung (1997) đã nhấn mạnh, rất phù hợp với nghiên cứu có hai hoặc nhiều hơn các tình huống nghiên cứu và đặc biệt giúp tìm ra ý nghĩa và nguyên nhân của khác biệt liên văn hóa dưới tác động của nhiều biến bối cảnh đa dạng.

\subsection{1. Đối tượng nghiên cúu}

Nghiên cứu khảo sát cách nói lời xin 1ỗi của 40 người gồm 20 ĐTKS người Mĩ đang làm việc tại Việt Nam, và 20 ĐTKS 
người Việt đang làm việc trong các tổ chức có đồng nghiệp là người Mĩ. Nhóm 20 ĐTKS Mĩ là những người sinh sống và tiếp xúc với đồng nghiệp là người Việt ít nhất từ 1 năm trở lên, ở đây họ được hiểu là những người Mĩ "thông thường”, nôm na là những người sinh ra tại Hoa Kỳ và tuân theo những giá trị xã hội và chuẩn mực văn hóa của nền văn hóa này. Mặc dù Hoa Kỳ được mệnh danh là "một quốc gia của các quốc gia”, không thể phủ nhận cho dù văn hóa có đa dạng nhưng để hòa nhập vào cuộc sống ở đây, những người Mĩ không kể gốc tộc chung sống và tuân thủ theo những quy luật văn hóa nhất định mà số đông người ta tuân thủ ở nền văn hóa này.

Nhóm đối tượng 20 ĐTKS Việt là những người làm việc trong các cơ quan, tổ chức có đồng nghiệp là người Mĩ, và sử dụng tiếng Anh như ngôn ngữ giao tiếp với đồng nghiệp Mĩ hàng ngày. Tiêu chí này đặt ra nhằm đảm bảo rằng người tham gia có một khả năng ngôn ngữ lưu loát và không gặp khó khăn trong việc diễn tả lời xin lỗi của họ bằng tiếng Anh trong ba tình huống đặt ra.

\subsubsection{Phiếu câu hỏi diễn ngôn (DCT)}

Phiếu câu hỏi diễn ngôn (DCT) mặc dù còn có hạn chế liên quan đến khả năng thu thập dữ liệu xác thực trong nghiên cứu văn hóa, mà theo một số học giả chỉ có thể được khắc phục thông qua công cụ ghi âm lời nói trong văn cảnh tự nhiên thông qua quan sát nhân chủng học (ethnographic observation) (Blum-Kulka, House \& Kasper, 1989, Manes \& Wolfson, 1981, xem thêm Billmyer \& Varghese, 1996, 2000), nhưng là một trong những công cụ thu thập dữ liệu phổ biến nhất được dùng trong các nghiên cứu về hành động lời nói từ trước đến nay. Một số nghiên cứu cụ thể đã sử dụng DCT bao gồm nghiên cứu về Cách xin lồi (Olshtain \& Cohen 1993; BlumKulka et. al; Linnel, Porter, Stone, \&Chen, 1992; Bergman \& Kasper, 1993), Thể hiện sụ biết on (Eisenstein \& Bodman, 1986, 1993), Cách tù chối (Beebe, Takahashi, \& UlissWeltzet, 1990; Bardovi-Harlig \& Hartford, 1991), Cách khuyên (Hinkel, 1997), và Cách yêu cầu (Blum-Kulka et al., 1989). Nói cách khác, đây là nguồn công cụ mà việc dạy, học và kiểm tra ngữ dụng học sẽ còn phụ thuộc vào nhiều (Billmyer \& Varghese, 1996, 2000).

Ba tình huống đưa ra trong phiếu câu hỏi diễn ngôn là:

Tình huống 1: Tại bãi đậu xe của công ty, bạn vô ý đi nhanh và va chạm một chiếc ô tô của đồng nghiệp đang chuẩn bị đỗ.

Tình huống 2: Sáng nay bạn đi làm trong tâm trạng không tốt vì đội bóng bạn hâm mộ bị thua tối qua. Một người đồng nghiệp đến hỏi bạn điều gì đó, nhưng trước khi kịp nhận ra họ, bạn không giữ được bình tĩnh và lớn tiếng với người đó.

Tình huống 3: Con bạn được sáu tuổi và hôm nay ở cơ quan tổ chức tiệc cuối năm, bạn đưa con đi theo. Tuy nhiên trong khi bạn đang bận trò chuyện với một vài đồng nghiệp, con bạn chẳng may huých phải tay một người đồng nghiệp làm đồ uống của họ đổ tràn lên chính trang phục của họ.

Trong ba tình huống đưa ra, ĐTKS trực tiếp gây lỗi ở tình huống 1 và tình huống 2 , trong khi đó ở ở tình huống 3 , người được khảo sát không trực tiếp gây lỗi. Tuy nhiên, cả ba tình huống đều được người tham gia đánh giá là yêu cầu lời xin lỗi trực tiếp trong giao tiếp giữa họ và các đồng nghiệp. 


\section{Kết quả và thảo luận}

\subsection{Tổng quan về CLXL}

Dữ liệu từ bảng câu hỏi thu thập từ hai nhóm đối tượng đã chỉ ra rằng hầu hết các chiến lược rơi vào sự kết hợp trong bản miêu tả CLXL của Cohen và Olstain (1993) và Trosborg (1995). CLXL hai nhóm khảo sát sử dụng được tổng hợp thành bảy chiến lược (CL1) Biểu đạt sụ xin lỗi, (CL2) Nhận trách nhiệm, (CL3) Giải thích lỗi, (CL4) Tối hiểu hóa lỗi, (CL5) Đề nghị đền bù/ sủa lỗi, (CL6) Hứa không tái phạm, và (CL7) Bày tỏ quan tâm được minh họa với các ví dụ dưới đây.

\section{Chiến lược 1: Biểu đạt sự xin lỗi}

CLXL này được sử dụng bởi phần lớn ĐTKS người Việt và người Mĩ

\begin{tabular}{l|l}
\multicolumn{1}{c|}{ Nhóm ĐTKS Việt } & \multicolumn{1}{|c}{ Nhóm ĐTKS Mĩ } \\
- Sir, I'm so sorry for & $\bullet$ I'm sorry if I was \\
being rude to you & abrupt \\
- I don't know how & $\bullet$ I apologize for being \\
to express my deep & so short with you \\
apology for this &
\end{tabular}

\section{Chiến lược 2: Nhận trách nhiệm}

CLXL này được toàn bộ ĐTKS Việt và 80\% ĐTKS Mĩ sử dụng

\begin{tabular}{l|l} 
Nhóm ĐTKS Việt & Nhóm ĐTKS Mĩ \\
- I shouldn't have let & $\bullet$ I shouldn't have taken \\
my child run around & out my frustration from \\
like that & my personal life on you \\
- No matter what & $\bullet$ My child doesn't \\
things happened to & know any better and \\
me, I don't have the & shouldn't have been \\
right to do so & running around
\end{tabular}

Chiến lược 3: Giải thích 1ỗi

CLXL này được xem là lời xin lỗi gián tiếp. Tất cả ĐTKS Việt và 90\% ĐTKS Mĩ sử dụng CL này

\begin{tabular}{|c|c|}
\hline Nhóm ĐTKS Viêt & Nhóm ĐTKS Mĩ \\
\hline - My child is so & - I'm overreacting \\
\hline playful & because I'm upset \\
\hline - It seems that I have & about something else \\
\hline been driving too fast & $\begin{array}{l}\text { - I'm a little upset } \\
\text { from last night's loss, } \\
\text { and it has set me on } \\
\text { edge today }\end{array}$ \\
\hline
\end{tabular}

Chiến lược 4: Tối thiểu hóa lỗi

CLXL này giúp giảm thiểu mức độ nghiêm trọng của lỗi, được 65\% ĐTKS Việt và $60 \%$ ĐTKS Mĩ sử dụng

\begin{tabular}{|c|c|}
\hline Nhóm ĐTKS Việt & Nhóm ĐTKS Mĩ \\
\hline must have kept & - I don't know how \\
\hline her firm at one & $\begin{array}{l}\text { this happened, but I } \\
\text { promise I'll let you }\end{array}$ \\
\hline $\begin{array}{l}\text { friend, if you } \\
\text { selled at by any }\end{array}$ & $\begin{array}{l}\text { drive into my car in } \\
\text { return }\end{array}$ \\
\hline me knon & - Oh, hi, if I'd known \\
\hline at hin & you were standing \\
\hline йль & there I would've \\
\hline o you & yell \\
\hline
\end{tabular}

Chiến lược 5: Đề nghị đền bù/ sửa lỗi

Có đến $3 / 4$ ĐTKS Mĩ sử dụng CLXL nhưng chỉ có 45\% ĐTKS Việt sử dụng CLXL này

\begin{tabular}{l|l} 
Nhóm ĐTKS Việt & \multicolumn{1}{|c}{ Nhóm ĐTKS Mĩ } \\
- I'll make up for & I will try to answer \\
all the expenses the & your question more \\
service costs you & calmly \\
- I will wash it up after & $\bullet$ Please giveme the bill \\
the party if you want & for the dry cleaning?
\end{tabular}


Chiến lược 6: Hứa không tái phạ

CLXL này chỉ được một ĐTKS Việt sử dụng (5\%) và bốn ĐTKS Mĩ sử dụng (20\%)

\begin{tabular}{l|l} 
Nhóm ĐTKS Việt & \multicolumn{2}{|c}{ Nhóm ĐTKS Mĩ } \\
- I will be more & $\begin{array}{l}\bullet \text { I will ensure it won't } \\
\text { careful next time. }\end{array}$ \\
happen again \\
- I promise I will not \\
do it next time & $\begin{array}{llr}\bullet \text { I'll have better } \\
\text { control over my } \\
\text { emotions next time }\end{array}$
\end{tabular}

Chiến lược 7: Bày tỏ quan tâm

100\% ĐTKS Việt trong khi chỉ có 40\% ĐTKS Mĩ sử dụng CLXL này

\begin{tabular}{l|l} 
Nhóm ĐTKS Việt & \multicolumn{1}{|c}{ Nhóm ĐTKS Mĩ } \\
- Are you injured? & - Are you OK? \\
- Is there anything & • You didn't get hurt, \\
wrong with you and & did you? \\
the car?
\end{tabular}

\subsubsection{Các CLXL được sủ dụng bởi nhóm} ĐTKS Việt

Bảng 1. CLXL của nhóm ĐTKS Việt

\begin{tabular}{|c|c|c|c|c|c|c|}
\hline \multirow{2}{*}{ CLXL } & \multicolumn{2}{|c|}{ Tình huống 1} & \multicolumn{2}{|c|}{ Tình huống 2} & \multicolumn{2}{|c|}{ Tình huống 3} \\
\hline & \begin{tabular}{c|} 
Số \\
lượng
\end{tabular} & $\%$ & \begin{tabular}{|c|} 
Số \\
lượng
\end{tabular} & $\%$ & $\begin{array}{c}\text { Số } \\
\text { lượng }\end{array}$ & $\%$ \\
\hline $\begin{array}{l}\text { 1. Biểu đạt } \\
\text { sụ xin lồi }\end{array}$ & 20 & $100 \%$ & 20 & $100 \%$ & 18 & $90 \%$ \\
\hline $\begin{array}{l}\text { 2. Nhận } \\
\text { trách nhiệm }\end{array}$ & 10 & $50 \%$ & 15 & $75 \%$ & 13 & $65 \%$ \\
\hline $\begin{array}{l}\text { 3. Giải } \\
\text { thich lối }\end{array}$ & 5 & $25 \%$ & 16 & $80 \%$ & 12 & $60 \%$ \\
\hline $\begin{array}{l}\text { 4. Tối thiểu } \\
\text { hóa lỗi }\end{array}$ & 6 & $30 \%$ & 7 & $35 \%$ & 6 & $30 \%$ \\
\hline $\begin{array}{l}\text { 5. Đề nghị } \\
\text { đền bù/ süa } \\
\text { lồi }\end{array}$ & 5 & $25 \%$ & 0 & $0 \%$ & 5 & $25 \%$ \\
\hline $\begin{array}{l}\text { 6. Húa } \\
\text { không tái } \\
\text { phạm }\end{array}$ & 1 & $5 \%$ & 1 & $5 \%$ & 10 & $50 \%$ \\
\hline $\begin{array}{l}\text { 7. Bày tỏ } \\
\text { quan tâm }\end{array}$ & 15 & $75 \%$ & 11 & $55 \%$ & 14 & $70 \%$ \\
\hline
\end{tabular}

Dữ liệu cho thấy hầu hết ĐTKS Việt sử dụng CL1 Biểu đạt sụ xin lỗi trong cả ba tình huống. Ở TH3 khi đối tượng nghiên cứu không trực tiếp gây lỗi, lời xin lỗi của hai ĐTKS không biểu đạt lời xin lỗi này. Cùng với CL1, chiến lược CL7 Bày tỏ quan tâm là chiến lược được sử dụng nhiều thứ hai $(75 \%$ ĐTKS Việt trong $T H 1,70 \%$ trong $T H 3$, và ít hơn một chút 55\% ở TH2).

Mặc dù có đến 55\% ĐTKS Việt sử dụng CL7 ở TH2, đây là tình huống mà người phạm lỗi lỡ lời to tiếng với đồng nghiệp, đối tượng nghiên cứu có xu hướng sử dụng CL2 Nhận trách nhiệm (75\%) và CL3 Giải thich lối $(80 \%)$ nhiều hơn các CLXL còn lại. TH2 là tình huống gây ảnh hưởng trực tiếp đến cảm xúc của đồng nghiệp, vì vậy các ĐTKS Việt muốn nhận trách nhiệm về mình và cố gắng giải thích rằng việc mình làm là không cố ý để giảm tổn thương đến đồng nghiệp.

Tần suất sử dụng CL5 Đề nghị đền bùl sưa chũa cũng thay đổi ở cả ba tình huống. Cụ thể, không ĐTKS Việt nào sử dụng CL5 ở $T H 2$ trong khi ở $T H 1$ và $T H 3$, lỗi gây ra liên quan đến tài sản của đồng nghiệp cho nên các ĐTKS cho rằng đề nghị đền bù là việc cần thiết. Tuy nhiên, số lượng ĐTKS Việt dùng $\mathrm{CL}$ này lại không hề cao. Có thể thấy rằng các dữ liệu cho thấy người Việt trong nghiên cứu này ưu tiên thể hiện sự quan tâm cá nhân đến đồng nghiệp hơn so với việc đề cập đến đền bù về tài chính cho họ.

Tương tự, CL6 Hía không tái phạm dù chỉ được sử dụng bởi một đối tượng nghiên cứu ở $T H 1$ và $T H 2$, nhưng có đến $50 \%$ số ĐTKS sử dụng CLXL này ở TH3. Trong trường hợp này, các ĐTKS đã sử dụng CLXL này để vừa dạy bảo cho con vừa cảnh báo để đứa trẻ không tái phạm. 
4.1.2. Các CLXL được sư dụng bởi nhóm ĐTKS Mĩ

Bảng 2: CLXL của nhóm ĐTKS Mĩ

\begin{tabular}{|l|c|c|c|c|c|c|}
\hline CLXL & \multicolumn{2}{|c|}{ Tình huống 1 } & \multicolumn{2}{|c|}{ Tình huống 2 } & \multicolumn{2}{|c|}{ Tình huống 3 } \\
\hline & SL & $\%$ & SL & $\%$ & SL & $\%$ \\
\hline $\begin{array}{l}\text { 1. Biểu đạt } \\
\text { sụ xin lỗi }\end{array}$ & 4 & $20 \%$ & 20 & $100 \%$ & 20 & $100 \%$ \\
\hline $\begin{array}{l}\text { 2. Nhận } \\
\text { trách } \\
\text { nhiệm }\end{array}$ & 6 & $30 \%$ & 14 & $70 \%$ & 7 & $35 \%$ \\
\hline $\begin{array}{l}\text { 3. Giải } \\
\text { thích lỗi }\end{array}$ & 0 & $0 \%$ & 17 & $85 \%$ & 3 & $15 \%$ \\
\hline $\begin{array}{l}\text { 4. Tối thiểu } \\
\text { hóa lồi }\end{array}$ & 12 & $60 \%$ & 4 & $20 \%$ & 4 & $20 \%$ \\
\hline $\begin{array}{l}\text { 5. Đề nghì } \\
\text { dền bù/ sửa } \\
\text { lối }\end{array}$ & 12 & $60 \%$ & 1 & $5 \%$ & 18 & $90 \%$ \\
\hline $\begin{array}{l}\text { 6. Hứa } \\
\text { không tái } \\
\text { phạm }\end{array}$ & 0 & $0 \%$ & 4 & $20 \%$ & 0 & $0 \%$ \\
\hline $\begin{array}{l}\text { 7. Bày tỏ } \\
\text { quan tâm }\end{array}$ & 7 & $35 \%$ & 0 & $0 \%$ & 4 & $20 \%$ \\
\hline
\end{tabular}

Bảng dữ liệu trên cho thấy tuy tồn tại khá nhiều khác biệt trong tần suất sử dụng các CLXL khác nhau ở ba TH, các ĐTKS Mĩ có sự tương đồng trong việc luôn luôn dùng $C L 1$ Biểu đạt sự xin lỗi khi xin lỗi đồng nghiệp ở $\mathrm{TH} 2$ và TH3. Dữ liệu cho thấy như vậy trong trong bất cứ trường hợp nào, việc tiên quyết cần làm là biết nói xin lỗi, đơn giản một câu ngắn gọn như "Tôi xin lỗi” (I'm sorry/ I do apologise) là đủ để đồng nghiệp của họ nhận biết rằng người gây lỗi nhận thức được lỗi mình gây ra.

Hai chiến lược CL6 Hưa không tái phạm và $C L 7$ Bày tỏ quan tâm được sử dụng với tần suất khá thấp, thậm chí CL6 không được sử dụng ở $T H 1$ và $T H 3$, còn $C L 7$ không được dùng ở TH2. Ở TH1, khi không may va chạm với phương tiện của người khác, câu trả lời của các ĐTKS Mĩ đồng nhất ở điểm họ không hấp tấp nhận trách nhiệm về mặt cá nhân hay mất công giải thích lý do. Thay vào đó, sự việc nên được giải quyết bởi hãng bảo hiểm xe. Một ĐTKS viết thêm trong phiếu trả lời rằng nếu đối tượng nhận lỗi là của mình, công ty bảo hiểm sẽ không chi trả cho thiệt hại hoặc họ sẽ phải trả phí bảo hiểm cao hơn, vì vậy tốt nhất không nên nhận lỗi. Vì vậy, trong TH1, hai CLXL được sử dụng phổ biến nhất CL4 Tối thiểu hóa lỗi và CL5 Đề nghị đền bù đều liên quan đến giải quyết vấn đề theo lý trí, và CL1 Biểu đạt sụ xin lỗi được rất ít người sử dụng (20\%).

CL5 Đề nghị đền bù cũng được sử dụng bởi nhiều ĐTKS Mĩ ở TH3 khi trang phục của đồng nghiệp bị vấy bẩn vì lỗi của con họ gây ra. Như vậy, với nhóm người Mĩ, có thể nói rằng khi có một tổn hại rõ ràng đến tài sản của người khác thì việc sử dụng CL5 Đề nghị đền bù phổ biến nhất. Ở TH3 là trường hợp thể diện của người đồng nghiệp có nguy cơ bị đe dọa cao, có thể vì vậy mà các ĐTKS Mĩ quyết định chọn CLXL này để kịp thời sửa chữa lỗi mà đứa trẻ gây ra, và cũng đồng thời để đứa trẻ nhận thức được hậu quả nghiêm trọng của sự bất cẩn vô ý của chúng.

Trong khi CL2 Nhận trách nhiệm không được sử dụng nhiều ở TH1 hay TH3, CLXL này lại được khá nhiều người Mĩ sử dụng ở TH2 (70\%) cùng với việc có đến 85\% ĐTKS người Mĩ sử dụng CL3 Giải thích lối kèm theo trong trường hợp này. Một vài ĐTKS giải thích thêm trong bản trả lời rằng việc lỡ lời với đồng nghiệp là hành động rất bất lịch sự, và vì vậy đồng nghiệp của họ xứng đáng nhận được lời giải thích cặn kẽ rằng họ bị oan. Cụ thể, một ĐTKS đã viết rằng họ sẽ ngay lập tức xin lỗi và giải thích rằng tâm trạng và hành vi tiêu cực của không hề liên quan đến đồng nghiệp dù với tư cách cá nhân hay trong công việc. 
Nói tóm lại, các CLXL được nhóm ĐTKS Mĩ sử dụng khá khác nhau ở ba tình huống. Trong khi phần lớn đối tượng nghiên cứu có khuynh hướng sử dụng CL4 Tối thiểu hóa lỗi và CL5 Đề nghị đền bù/ sưa lối ở TH1 (60\%), ở TH2, họ lại chọn sử dụng CL2 Nhận trách nhiệm và CL3 Giải thích lỗi. Cuối cùng, trong TH3, CL5 Đề nghị đền bù là chiến lược được sử dụng nhiều nhất bởi 18 ĐTKS.

\subsection{Các CLXL sủ dụng trong ba tình huống}

\subsubsection{Các CLXL trong tình huống 1}

Bảng 3. CLXL sử dụng bởi hai nhóm trong tình huống 1

\begin{tabular}{|c|c|c|c|c|c|c|c|}
\hline CLXL & CL1 & CL2 & CL3 & CL4 & CL5 & CL6 & CL7 \\
\hline Ng.Việt & $100 \%$ & $50 \%$ & $25 \%$ & $30 \%$ & $25 \%$ & $5 \%$ & $75 \%$ \\
\hline Ng.Mĩ & $20 \%$ & $30 \%$ & $0 \%$ & $60 \%$ & $60 \%$ & $0 \%$ & $35 \%$ \\
\hline
\end{tabular}

Ở TH1, hai CLXL người Mĩ sử dụng nhiều hơn so với nhóm ĐTKS Việt là CL4 Tối thiểu hóa lỗi và CL5 Đề nghi đền bù/ sưa lỗi. Số lượng ĐTKS Mĩ sử dụng hai CL này đều cao gấp đôi hoặc hơn so với số lượng ĐTKS Việt sử dụng chúng.

Ngược lại, ở năm CLXL còn lại, trừ CL6 Hưa không tái pham, số lượng người Việt sử dụng các CLXL còn lại đều cao hơn nhiều so với ĐTKS Mĩ, cụ thể trong khi có tới 75\% ĐTKS Việt sử dụng CL7 Bày tỏ quan tâm hay 100\% sử dụng CL1 Biểu đạt sụ xin lỗi thì chỉ có lần lượt $35 \%$ và $20 \%$ ĐTKS Mĩ sử dụng hai CLXL này.

\subsubsection{Các CLXL trong tình huống 2}

Bảng 4. CLXL sử dụng bởi hai nhóm trong tình huống 2

\begin{tabular}{|c|c|c|c|c|c|c|c|}
\hline CLXL & CL1 & CL2 & CL3 & CL4 & CL5 & CL6 & CL7 \\
\hline Ng.Việt & $100 \%$ & $75 \%$ & $80 \%$ & $35 \%$ & $0 \%$ & $5 \%$ & $55 \%$ \\
\hline Ng.Mĩ & $100 \%$ & $70 \%$ & $85 \%$ & $20 \%$ & $5 \%$ & $20 \%$ & $0 \%$ \\
\hline
\end{tabular}

Ở TH2, điểm tương đồng giữa hai nhóm ĐTKS là họ đều sử dụng ba chiến lược $C L 1$
Biểu đạt sụ xin lỗi, CL2 Nhận trách nhiệm, và CL3 Giải thích lỗi nhiều nhất khi lỡ to tiếng với đồng nghiệp vì lí do cá nhân của bản thân.

Một điểm tương đồng khác là ở cả hai nhóm đều có một CLXL không được sử dụng, mặc dù ở nhóm ĐTKS Việt, đó là CL5 Đề nghị đền bù/ sứa lối, trong khi đó là CL7 Bày tỏ quan tâm đối với nhóm ĐTKS Mĩ. Trong khi có đến $55 \%$ người Việt bày tỏ sự quan tâm đến tâm trạng của đối phương trước, thì nhóm ĐTKS Mĩ lại ưu tiên nhận trách nhiệm cá nhân và hứa không tái phạm. CL4 Tối thiểu hóa lỗi không được sử dụng quá nhiều, chỉ khoảng $1 / 4$ đến $1 / 3$ số lượng ĐTKS sử dụng CLXL này trong $\mathrm{TH} 2$.

\subsubsection{Các CLXL trong tình huống 3}

Trong TH3, người tham gia nghiên cứu không là người trực tiếp gây ra lỗi, vì vậy trong bản câu hỏi khảo sát, tác giả đã hỏi thêm một câu để xem rằng liệu trong trường hợp này, họ sẽ (1) xin lỗi đồng nghiệp trước rồi mới yêu cầu đứa trẻ xin lỗi, hay (2) sẽ mặc định rằng đứa trẻ tự biết xin lỗi trước rồi họ mới xin lỗi đồng nghiệp. Kết quả cho thấy rằng, trong khi 100\% nhóm người Việt lựa chọn phương án 1 là xin lỗi đồng nghiệp trước khi yêu cầu con mình xin lỗi họ, thì phần lớn người Mĩ (17/20 đối tượng) lại chọn phương án là yêu cầu con mình xin lỗi trước sau đó họ sẽ xin lỗi đồng nghiệp. Một trong số 17 ĐTKS Mĩ đó chia sẻ rằng một đứa trẻ sáu tuổi cần biết nhận trách nhiệm về những gì mình làm, và vì vậy phải biết kịp thời xin lỗi người đồng nghiệp của bố mẹ trong tình huống này.

Sự khác nhau trong cách giải quyết tình huống này đã phần nào thể hiện được sự khác biệt trong cách suy nghĩ và giao tiếp của hai nhóm đến từ hai nền văn hóa Việt và Mĩ. Như đã đề cập ở phần nội dung lý luận, với tính 
cộng đồng cao, câu trả lời của nhóm ĐTKS người Việt trong nghiên cứu này đã phản ánh được nhận định của Mạc Giao (2002) rằng người làm cha mẹ và con cái luôn có một sự gắn kết và lỗi con gây ra thì cha mẹ cũng có phần trách nhiệm. Trái lại, văn hóa Mĩ tôn trọng tính cá nhân được phản ánh rõ nét qua lựa chọn CLXL của nhóm đối tượng người Mĩ khi dữ liệu chỉ ra rằng tại bữa tiệc, đứa trẻ cần phải nhận thức được và có trách nhiệm với hành động cá nhân của bản thân, và vì vậy cần biết nói lời xin lỗi khi gây lỗi mà không cần phải chờ để bố mẹ nhắc nhở hay yêu cầu.

Để tìm hiểu thêm về việc sử dụng CLXL, các ĐTKS được yêu cầu đưa ra lời xin lỗi của mình trong tình huống này, và các CLXL họ sử dụng được tóm tắt ở bảng sau:

Bảng 5. CLXL sử dụng bởi hai nhóm trong tình huống 3

\begin{tabular}{|l|l|l|l|l|l|l|l|}
\hline CLXL & $C L 1$ & $C L 2$ & $C L 3$ & CL4 & CL5 & CL6 & CL7 \\
\hline Ng.Việt & $90 \%$ & $65 \%$ & $60 \%$ & $30 \%$ & $25 \%$ & $50 \%$ & $70 \%$ \\
\hline Ng.Mĩ & $100 \%$ & $35 \%$ & $15 \%$ & $20 \%$ & $90 \%$ & $0 \%$ & $20 \%$ \\
\hline
\end{tabular}

CL1 Biểu đạt lời xin lỗi mặc dù vẫn là CLXL được sử dụng nhiều nhất so với sáu CLXL còn lại, nhưng trong TH3 chỉ được sử dụng bởi 18 trên tổng số 20 người Việt. Điều này khác với hai TH1 và TH2 khi mà $100 \%$ nhóm này sử dụng CL1 ở hai tình huống trước.

Một điểm khác biệt cơ bản giữa việc lựa chọn CLXL của hai nhóm là nhóm ĐTKS Việt có xu hướng sử dụng CL3 Giải thích lỗi, CL6 Hứa không tái phạm và $C L 7$ Bày tỏ quan tâm trong trường hợp này cao hơn nhiều so với các đối tượng người Mĩ. Ví dụ, nhiều ĐTKS Việt cho rằng việc bày tỏ sự quan tâm của họ đến đồng nghiệp trong trường hợp này là cần thiết và cần được thực hiện trước tiên sau lời xin lỗi. Tuy nhiên, nhiều ĐTKS Mĩ lại cho rằng họ cần lập tức đưa ra cách giải quyết tình huống, bằng việc rất nhiều người trong số họ $(18 / 20)$ thấy cần đưa ra việc đền bù thiệt hại (CL5 Đề nghi đền bù/sưa lỗi) cho chiếc áo/ váy bị vấy bẩn của người đồng nghiệp. Họ cho rằng trong trường hợp này trang phục cần được giặt khô để tẩy vết bẩn, và vì vậy cần đền bù hóa đơn giặt cho đồng nghiệp.

\section{Kết luận}

Kết quả thu được của nghiên cứu này chỉ rằng khi xin lỗi bằng ngôn ngữ Anh, hai nhóm đối tượng nghiên cứu người Việt và người Mĩ có khá nhiều điểm tương đồng trong việc lựa chọn CLXL nhưng ở các tình huống khác nhau, tần suất sử dụng các CLXL mang nhiều khác biệt.

Trước tiên, cả hai nhóm Việt và Mĩ đều có mức độ sử dụng $C L 1$ Biểu đạt lời xin lỗi cao và CL2 Nhận trách nhiệm tương đối cao hơn so với bốn CLXL còn lại, điều này trùng với kết luận của một vài nghiên cứu trước đây của Cohen và Olshtain (1993), Linnel và cộng sự (1992), Trosborg (1995), Phuong (2000) and Van (2000). Dữ liệu thu được qua ba tình huống về các lỗi khác nhau phần nào cũng phản ánh được nhận định của học giả Olshtain (1989) và Elli (1994) rằng trong các trường hợp khác nhau bất kể mức độ nghiêm trọng của lỗi hay ảnh hưởng của các biến nội ngoại cảnh, các CLXL được nhiều nhóm đối tượng sử dụng dường như có những tương đồng nhất định. Tuy nhiên, kết quả nghiên cứu cũng chỉ ra được những điểm dị biệt trong việc lựa chọn CLXL giữa hai nhóm người Mĩ và người Việt. CLXL mà người Mĩ sử dụng nhiều nhất trong ba tình huống đặt ra lại là CL5 Đề nghi đền bù/sưa lỗi trong khi nhóm ĐTKS Việt nghiêng về CL7 Bày tỏ quan tâm tới khi nói lời xin lỗi. Việc rất ít người Việt sử dụng CL5 
Đề nghi đền bù/sủa lỗi trong ba tình huống phần nào khơi lại kết luận trong vài nghiên cứu trước đây của Cohen và Olshtain (1993), Trosborg (1987, 1995), hay của Bergman và Kasper (1993) rằng trong $\mathrm{HVXL}$, một vài CLXL chỉ xuất hiện trong cách xin lỗi của người bản ngữ nói tiếng Anh mà không hề được sử dụng bởi nhóm ĐTKS sử dụng tiếng Anh như ngoại ngữ.

Kết quả nghiên cứu về những tương đồng và khác biệt trên cho thấy việc lựa chọn các CLXL của hai nhóm khảo sát phần nào phản ánh được về một nền văn hóa Việt Nam có tính cộng đồng cao và trọng tính hòa thuận trong giao tiếp xã hội, trong mối tương quan so sánh với nền văn hóa Mĩ nhấn mạnh trách nhiệm của từng cá nhân trong xã hội và ưu tiên giải quyết các sự việc theo lý trí (Hofstede, 1980). Việt Nam được coi là nền văn hóa trọng tình cảm (Hofstede, 1980) và ở đó việc duy trì sự hòa thuận trong giao tiếp luôn được đặt lên hàng đầu cho nên khi phạm lỗi, điều mà người Việt hay làm là bày tỏ sự quan tâm của mình đến đối phương bị hại, đặc biệt là trong các tình huống đe dọa thể diện của người liên quan. Ngược lại, trong văn hóa Mĩ, tính lý trí là trọng tâm, vì vậy người Mĩ trong nghiên cứu này thay vì bày tỏ sự quan tâm theo hướng tình lại thiên về giải quyết các tình huống phạm lỗi theo lý. Họ ưu tiên giải quyết tình huống thông qua cách nhận lỗi và giải quyết lỗi trực tiếp, hướng đền việc đền bù tổn hại của đồng nghiệp. Ngoài ra, tình huống 3 phần nào thể hiện được một điểm khác biệt trong hai nền văn hóa: đó là tính tập thể cao của người Việt và tính cá nhân cao của người Mĩ. Cụ thể, phần lớn các ĐTKS Mĩ tin rằng mỗi cá nhân trong xã hội phải tự chịu trách nhiệm với hành động của mình trong khi với một nền văn hóa tính tập thể cao như Việt
Nam, thể diện cá nhân gắn liền với thể diện tập thể thì khi con mình gây lỗi, cha mẹ coi rằng "Con dại cái mang", lỗi của con cũng là lỗi của cha mẹ chưa biết dạy bảo cẩn thận, và coi rằng họ là người lớn tuổi hơn nên sẽ xin lỗi đồng nghiệp trước khi con họ thực hiện HVXL. Cuối cùng, dữ liệu về việc sử dụng các CLXL đã chỉ ra rằng, với các ĐTKS trong nghiên cứu này, mặc dù có thời gian làm việc chung với đồng nghiệp Mĩ nhưng cách người Việt hay người Mĩ xin lỗi bằng tiếng Anh vẫn chịu ảnh hưởng của văn hóa giao tiếp gốc.

\section{Tài liệu tham khảo}

\section{Tiếng Việt}

Nguyễn Quang (1998), Một số khác biệt giao tiếp lời nói Việt - Mỹ trong cách thức khen và tiếp nhận lời khen . Luận án tiến sĩ khoa học ngữ văn. ĐHKHXH\&NV

\section{Tiếng Anh}

Bardovi-Harlig, K. \& Hartford, B. S. (1991). Saying "no" in English: Native and nonnative rejections. In L. F. Bouton (Ed.), Pragmatics and Language Learning, 2, 41-57. Retrieved September 7, 2016 from www.carla.umn. edu/speechacts/refusals/structure.html

Beebe, L., Takahashi, T., \& Uliss-Weltz, R. M. (1990), Pragmatic Transfer in the ESL Refusals. Retrieved September 7, 2016 from www.carla.umn.edu/ speechacts/refusals/ structure.html

Bergman, M. \& Kasper, G. (1993). Perception and performance in native and nonnative apology. In G. Kasper \& S. Blum-Kulka (Eds), Interlanguage Pragmatics, Oxford: OUP.

Billmyer, K., \& Varghese, M. (1996). Investigating the structure of discourse completion tests, Working Papers in Educational Linguistics, Philadelphia: University of Philadelphia. 
Billmyer, K., \& Varghese, M. (2000). Investigating instrument-based pragmatic variability: Effects of enhancing discourse completion tests. Applied Linguistics, 21(4), 517-552.

Blum-Kulka, S., House, J. \& Kasper, G. (1989). Investigating cross-cultural pragmatics: An introductory overview. In S. Blum-Kulka, J. House \& G. Kasper (Eds), Cross-cultural pragmatics: Requests and apologies, 1-34, Norwood: Ablex Publishing.

Brown, P. \& Levinson, S. (1987). Politeness, Cambridge: Cambridge University Press.

Brown, P., \& Levinson, S. (1978). Universals of language usage: Politeness phenomena. In E. Olshtain (1989). Apologies across languages.

Cohen, A. D. \& Olshtain, E. (1993). The production of speech acts by EFL learners, TESOL Quarterly, 27, 33-56.

Cohen, A. D., Olshtain, E., \& Rosenstein, D. S. (1986). Advanced EFL apologies: What remains to be learned? International Journal of the Sociology of Language, 62 (6), 51-74. Retrieved September 8, 2016 from http:// www.carla.umn.edu/speechacts/apologies

Dang Thanh Phuong (2000). A cross-cultural study of apologizing and responding to apologies in Vietnamese and English. Unpublished M.A. Thesis, Hanoi: CFL, VNU, Hanoi.

Do Thi Mai Thanh (2000). Some EnglishVietnamese cross-cultural differences in requesting. Unpublished MA Thesis Linguistics, Hanoi: CFL, VNU, Hanoi.

Eisenstein, M. \& Bodman, J.W. (1986). "I very appreciate": Expressions of gratitude by native and nonnative speakers of American English, Applied Linguistics 7 (2), 167-185.

Eisenstein, M. \& Bodman, J.W. (1993). Expressing gratitude in American English. In G. Kasper \& S. Blum-Kulka (Eds), Interlanguage Pragmatics, Oxford: Oxford University Press.
Ellis, R. (1994). The studies of second language acquisition. In A.S. Byon (2005), Apologizing in Korean: Cross-cultural Analysis in Classroom Settings, Korean Studies, 29, 137-166.

Garcia, C. (1989). Apologizing in English: Politeness strategies used by native speakers and non-native speakers, Multilingua 11, 387-406.

Geertz, C. (1973). The interpretation of cultures. In W.B. Gudykunst \& S. Ting-Toomey (1988), Culture and interpersonal communication, 2730, The US: Sage.

Gorlanes, G.J. and Brilhart, J.K. (1997). Communication in group ( $3^{\text {rd }}$ Ed.), The US: The McGraw-Hill, INC.

Gudykunst, W. B., Matsumoto, Y., Ting-Toomey, S., Nishida, T., Kim, K. S., \& Heyman, S. (1996). The influence of cultural individualismcollectivism, self construals, and individual values on communication styles across cultures. Human Communication Research, 22, 510-543.

Hall, E. (1976). Beyond culture, New York: Doubleday.

Hinkel, E. (1997). Appropriateness of advice: DCT and Multiple Choice Data, Applied Linguistics, 18 (1), 1-26.

Hofstede, G. (1980). Culture's consequences: International differences in work-related value, CA: Sage.

Kasper, G. (1996). Politeness, Handbook of Pragmatics, Amsterdam: Verschueren.

Kieu Thi Hong Van (2000). Apologies in English and Vietnamese. Unpublished M.A. Thesis, Hanoi: CFL, VNU, Hanoi.

Kieu Thi Thu Huong (2006). Disagreeing in English and Vietnamese: A pragmatics and conversation analysis perspective, Ph.D. Dissertation, Hanoi: CFL, VNU, Hanoi. 
Linnell, J., Porter, F. L., Stone, H., \& Chen, W. (1992). Can you apologize me? An investigation of speech act performance among non-native speakers of English. Working Papers in Educational Linguistics, 8, 33-53.

Lustig, M.W., \& Koester, J. (2006). Intercultural competence $\left(6^{\text {th }}\right.$ ed. $)$, Allyn \& Bacon: Pearson, The US.

Mac Giao (2002). A new approach to Vietnamese culture. Retrieved October 22, 2016, from http://www.dunglac.net/macgiao/vh-06-ketthumuc. $\mathrm{htm}$

Maeshiba, N., Yoshinaga, N., \& Kasper, G., \& Ross, S. (1996). Transfer and proficiency in interlanguage apologizing. In S. M. Gass \& J. Neu (Eds), Speech act across cultures challenges to communication in a second language, 155-187, New York: Mouton Du Gruyter.

Nguyen Phuong Suu (1990). A cross-cultural study of greeting and address terms in English and Vietnamese. Unpublished MA Thesis Linguistics, Australia: University of Canberra, Australia.

Nguyen Quang (2006). Lecture-notes on Crosscultural Communication, Hanoi: CFL, VNU, Hanoi.

Phan Thi Van Quyen (2001). Some EnglishVietnamese cross-cultural differences in refusing a request. Unpublished MA Thesis Linguistics, Hanoi: CFL, VNU, Hanoi

Rose, K. R. (2000). An exploratory crosssectional study of interlanguage pragmatic development. Studies in Second Language Acquisition, 22(1), 27-67.
Smith, Jr, Esmond, D \& Pham, C (1996). Doing business in Vietnam: A cultural guide, Business Horizons, 39 (3), 47-52

Stella T. (1992). Cross-Cultural Face-Negotiation: An Analytical Overview, Simon Fraser University.

Sugimoto, N. (1998). Norms of apology depicted in U.S. American and Japanese literature on manners and etiquette, International Journal of Intercultural Relations, 22 (3), 251-276.

Trice, H., \& Beyer, J. (1992). The cultures of work organizations, New York: Prentice-Hall.

Trosborg, A. (1987). Apology strategies in natives/nonnative. Pragmatics, 11, 147-167.

Trosborg, A. (1995). The communicative act of apologizing. In A. Trosborg, Interlanguage pragmatics: Requests, Complaints, Apologies, 373 - 433, New York: Mouton De Gruyter.

Van de vijver, F.J.R., \& Leung, K. (1997), Methods and Data Analysis for Cross-cultural Research. Newbury Park: Sage.

Wolfson, N., Marmor, T., \& Jones, S. (1989). Problems in the comparison of speech acts across cultures. In S. Blum-Kulka, J. House, \& G. Kasper (Eds.), Cross-cultural pragmatics: Requests and apologies, 174-196, Norwood: Ablex Publishing.

Yule, G. (1997). Pragmatics, Oxford: Oxford University Press. 


\title{
APOLOGIZING STRATEGIES BY VIETNAMESE SPEAKERS OF ENGLISH AND AMERICAN SPEAKERS OF ENGLISH
}

\author{
Nguyen Thuy Trang \\ Faculty of Linguistics and Cultures of English-speaking Countries, \\ VNU University of Languages and International Studies, Pham Van Dong, Cau Giay, \\ Hanoi, Vietnam
}

\begin{abstract}
This research has investigated how twenty American working with Vietnamese and twenty Vietnamese working with Americans in Vietnam performed the speech act of apologizing in three given situations. A discourse completion task was employed to investigate their choices of apologizing strategies in light of apologizing strategies proposed by Cohen and Olshtain (1993) and Trosborg (1995). Findings of the apologizing strategy preferences have, firstly, strengthened the arguments of previous cross-cultural studies by Olshtain (1989) and Elli (1994) that apologizing strategies tend to be similar cross-linguistically when comparable contexts and the level of offense are given. Nonetheless, the most distinguishable difference in the use of apologizing strategies by the participants is that the Americans often employed the strategy Offer of repair whereas Vietnamese subjects were more likely to show their Concern for the hearer through which the images of a sentimental Vietnamese culture and a rational American culture were sharpened. The features of individualism and collectivism were also found helpful in explaining this difference and above all, inspite of frequent contacts with one another, both the Vietnamese and American subjects were not affected by others' communicating practices.
\end{abstract}

Keywords: apologizing strategies, American culture, Vietnamese culture, speech act 